\title{
The paternal epigenome and embryogenesis: poising mechanisms for development
}

\author{
Timothy G Jenkins ${ }^{1}$ and Douglas T Carrell ${ }^{1,2,3}$
}

The scope of paternal contributions during early embryonic development has long been considered limited. Dramatic changes in chromatin structure throughout spermatogenesis have been thought to leave the sperm void of complex layers of epigenetic regulation over the DNA blueprint, thus leaving the balance of that regulation to the oocyte. However, recent work in the fields of epigenetics and male factor infertility has placed this long-held, and now controversial dogma, in a new light. Elegant studies investigating chromatin and epigenetic modifications in the developing sperm cell have provided new insights that may establish a more critical role for the paternal epigenome in the developing embryo. DNA methylation, histone tail modifications, targeted histone retention and protamine incorporation into the chromatin have great influence in the developing sperm cell. Perturbations in the establishment and/or maintenance of any of these epigenetic marks have been demonstrated to affect fertility status, ranging in severity from mild to catastrophic. Sperm require this myriad of chromatin structural changes not only to serve a protective role to DNA throughout spermatogenesis and future delivery to the egg, but also, it appears, to contribute to the developmental program of the future embryo. This review will focus on our current understanding of the epigenetics of sperm. We will discuss sperm-specific chromatin modifications that result in genes essential to development being poised for activation early in embryonic development, the disruption of which may result in reduced fecundity.

Asian Journal of Andrology (2011) 13, 76-80; doi:10.1038/aja.2010.61; published online 25 October 2010

Keywords: chromatin; DNA methylation; embryogenesis; epigenetics; histone modification; male infertility

\section{INTRODUCTION}

Mature mammalian spermatozoa are unique and highly specialized cells. The utility of this specialization is to facilitate the safe delivery of competent paternal DNA to the oocyte by providing protection from damage and ensuring that the DNA is informative to the future embryo. Any perturbations in epigenetic modifications or in mechanisms protecting DNA from fragmentation will result in either high levels of DNA damage or in a chromatin structure that is incapable of eliciting targeted gene activation in the embryo on its own. The result of either scenario is a germ cell with reduced fertilization capacity and/or decreased overall viability. If these perturbations are common among an entire sperm population, then the affected individual will likely have reduced fertility. ${ }^{1-8}$ These mechanisms, as well as others, result in the high numbers of male factor infertility cases that we see today. Although most cases are left defined as idiopathic, we are beginning to gain a better understanding of how epigenetic changes in the sperm may have a causative role in the etiology of infertility.

Robust paternal epigenetic contribution to embryogenesis requires that DNA, and chromatin structure as a whole, contain layers of regulatory elements that are sufficient to drive genes towards activation or silencing upon delivery to the egg. These epigenetic marks can include selective histone retention, histone tail modifications and DNA methylation.
Mature sperm contain a transcriptionally silent chromatin that is susceptible to DNA damage from outside agents. This damage can be catastrophic to the mature sperm as it lacks sufficient repair mechanisms. To avoid this damage, DNA undergoes dramatic chromatin structural rearrangements during the late stages of spermatogenesis, facilitated by different classes of nuclear proteins including histones, transition proteins, and protamines 1 and 2 (P1 and P2). The once 'loose' (nucleosome-bound) chromatin becomes tightly compacted via the stepwise replacement of histones with protamines. First, transition proteins replace the majority of canonical histones. Then, transition proteins are exchanged for protamines, which form tight toroid structures once integrated in the DNA. The resulting compacted chromatin structure protects against DNA damage and also silences transcription, as no polymerase machinery is able to gain sufficient access to the DNA. ${ }^{9-11}$

The level of DNA protection that chromatin compaction provides to the sperm seems to be in direct opposition to the cell's role in facilitating the delivery of competent DNA that is poised for activation at key genes essential in early embryonic development. The transition of nucleosome-bound DNA to protamine-bound DNA effectively removes a layer of epigenetic regulation from chromatin, as most histones are replaced by protamines, which induce a quiescent transcriptional status. The loss of this layer of regulation was thought to leave paternal DNA with little to contribute to the developing embryo

${ }^{1}$ Andrology and IVF Laboratories, Department of Surgery, University of Utah, Salt Lake City, UT 84108, USA; ${ }^{2}$ Department of Obstetrics and Gynecology, University of Utah School of Medicine, Salt Lake City, UT 84108, USA and ${ }^{3}$ Department of Physiology, University of Utah School of Medicine, Salt Lake City, UT 84108, USA

Correspondence: Dr D T Carrell (douglas.carrell@hsc.utah.edu)

Received: 21 July 2010; Revised: 18 August 2010; Accepted: 23 August 2010; Published online: 25 October 2010 
aside from the genome itself. However, studies are beginning to demonstrate how highly specialized and unique modifications retained in sperm chromatin may actually provide significant influence in the early embryo.

This review will focus on recent studies that have increased our understanding of how the mature sperm are able to facilitate the delivery of competent DNA to the oocyte and what the implications of this delivery may be in the developing embryo. We will also discuss the role of aberrant epigenetic profiles and their possible involvement in many cases of idiopathic male factor infertility.

\section{DNA METHYLATION}

DNA methylation is uniquely regulated in the germ cell to have important roles in the development of gametes as well as in the developing embryo. This epigenetic regulation is found directly on the DNA at cytosine residues, generally within cytosine-phosphate-guanine dinucleotides, and is regulated by the DNA methyltransferase (DNMT) family of proteins. ${ }^{12}$ The regulatory effect of DNA methylation is to decrease access of polymerase machinery to hypermethylated promoter regions at specific genes. Lack of methylation marks (hypomethylation) effectively places the gene in an inducible state for activation, thus promoting transcription.

The DNMT protein family includes both catalytic members responsible for laying down new methylation marks, as well as proteins responsible for the maintenance of those marks. DNMT3a, DNMT3b and DNMT3l work in concert to mediate de novo methylation in mammalian sperm, with DNMT3a and DNMT3b being responsible for catalyzing the methylation reaction. DNMT31 lacks a catalytic domain, yet is still essential in methylation establishment, as it facilitates DNMT3a and DNMT3b's actions and coordinates proper placement of marks. ${ }^{13}$ DNMT1 is the most predominate methyltransferase seen in somatic tissues and also lacks catalytic capabilities. Instead, it primarily facilitates proper maintenance of previously established methylation patterns during DNA replication before cell division. ${ }^{14}$ Without the establishment and maintenance of DNA methylation, fertilization can occur, but the embryo is unable to develop properly and, depending on the severity, will often result in arrest. $^{15}$

DNA methylation has proven to be of great importance in the regulation of gene expression in somatic cells, but what is its role in embryonic development? Many studies have demonstrated the essential role of DNA methylation in the developing embryo at the single gene, chromosome and whole genome level. One of the most wellstudied examples of this is genomic imprinting, which is primarily regulated by DNA methylation and is essential in ensuring a parent of origin gene expression pattern at imprinted loci. The embryo requires this type of gene silencing for normal development. ${ }^{16}$ Other important regulatory processes in the embryo require DNA methylation, including X chromosomal inactivation and retrotransposon silencing. These mechanisms ensure silencing of portions of the genome that, if expressed, could result in inappropriate regulation of transcription and in abnormal cell function. ${ }^{17-19}$ Whole-genome methylation status in the embryo has also proven to be of great importance to proper development ${ }^{17,20,21}$ and may be indicative of proper development and overall viability, although more work must be done to establish this. The most striking evidence for the role of DNA methylation in the developing embryo is that of targeted studies involving the perturbation of DNMT protein family members and the resulting phenotypes.

\section{DNA METHYLATION ABNORMALITIES, EMBRYOGENESIS AND INFERTILITY}

All members of the DNMT family are important for proper establishment and maintenance of methylation marks in developing cells. Disruption of these proteins clearly results in aberrant DNA methylation profiles globally as well as at specific genes. Utilizing targeted genetic knockout models as a technique for studying DNA methylation has afforded us insight into how methylation status can confer great influence over gene regulation and embryo viability.

DNMT1 knockouts and loss of function mutations in mice result in embryos with less than $5 \%$ of their normal methylation marks globally. This lack of methylation over the whole genome results in specific defects: imprinted genes show biallelic expression due to lack of silencing at imprinted loci, expression of retrotransposons and chromosome inactivation is halted. These embryos show retarded gestational growth and do not result in viable offspring. ${ }^{15,18,19}$

Disruption of de novo DNMTs (DNMT3a and DNMT3b) and the supporting DNMT3l proteins have also been demonstrated to affect fertility. Gene targeting by conditional knockout in the germ cell line (somatic cells were unaffected) of both DNMT3a and DNMT31 individually resulted in a marked hypomethylation at imprinted loci. ${ }^{22}$ The resultant mice were infertile and demonstrated a significant decrease in spermatogenesis. DNMT31 knockouts were also found to have global hypomethylation, ultimately resulting in decreased spermatogenesis and reduced fertility status. ${ }^{1,22}$ The disruption of DNMT3b did not result in the same phenotype; in fact, no unique phenotype was observed. However, the loss of DNMT3b did result in the loss of methylation at a single, specific locus (Rasgrf1) that was not seen in the DNMT3a conditional knockouts. ${ }^{22,23}$ These results suggest that there is likely much redundancy for DNMT3b function, but that there are likely specific genomic regions in which it may work independently. $^{23}$

Alterations of DNA methylation patterns on the paternal genome have been investigated in multiple studies utilizing 5-azacytidine and 5-aza-2'-deoxycytidine. Both these drugs inhibit DNA methylation and are commonly used in methylation studies. ${ }^{24}$ Treatment of both rats and mice with these DNA methylation inhibitors gave rise to multiple disease states in the affected animals. A marked reduction in overall fertility was seen in animals that were treated with shortterm exposure. ${ }^{25}$ In animals that underwent long-term exposure, more severe phenotypes were seen and correlates were established between DNA methylation status and embryo lethality. In addition, administration of 5-aza- $2^{\prime}$-deoxycytidine in mice resulted in decreased fertilization rates as well as an increased incidence of preimplantation loss. ${ }^{26-28}$ The use of these drugs has proven to reduce methylation status globally in germ cells, but the mechanism of effect on embryo lethality may also be a result of cytotoxic effects of the drug itself. $^{26}$ To verify the role of germ cell DNA methylation status and its effects on embryogenesis, more work with genetic knockout studies observing alterations in liter size, post-implantation loss and recurrent miscarriage as end points could be utilized.

Taken together, these data suggest that aberrant methylation in the male germ cell results in abnormal embryogenesis, decreased fertilization ability and an overall decrease in fertility status of affected individuals. For DNA methylation to facilitate gene regulation in the embryo, there must be differential methylation throughout the paternal genome, with hypomethylation at the promoter regions of important early embryonic developmental genes. This idea is supported by recent data, suggesting that methylation marks help to ensure that proper genes are poised for activation in the early embryo. ${ }^{29}$ Adding 
strength to this argument is a recent study evaluating sperm DNA methylation status in in vitro fertilization patients, which demonstrated that global hypomethylation was significantly correlated with poor pregnancy outcomes. ${ }^{30}$ However, further analysis and additional studies are required to draw clear correlates to embryogenesis.

\section{HISTONE TO PROTAMINE TRANSITION}

The chromatin structure of the mature sperm is tightly compacted as a result of dramatic structural rearrangements that occur during spermatogenesis. As mentioned earlier, the original histone-bound chromatin structure is replaced with protamine proteins through a stepwise process. First, canonical histones are replaced by transition proteins, which are then replaced by protamines (P1 and $\mathrm{P} 2)$. The incorporation of these proteins into the DNA is tightly regulated and results in an approximately $1: 1$ ratio of $\mathrm{P} 1$ to $\mathrm{P} 2 .^{2,9-11}$ This transition creates a chromatin structure that is between 6 and 20 times more compact than histone-bound DNA. ${ }^{31,32}$ The result is transcriptionally silent paternal DNA that is effectively protected against DNA damage.

Throughout the structural changes seen in spermatogenesis, there still remain a small portion of histones that are not replaced, including testes-specific histone variants and canonical histones. ${ }^{31}$ The retention of these histones could be either a result of inefficient machinery or because of some regulatory mechanism. Interestingly, recent studies have found this histone retention to be programmatic in nature. Nucleosome-bound DNA is retained at regions of high genetic importance for the early embryo: developmental gene promoters, imprinted loci and microRNA clusters. ${ }^{29}$ As one would expect, any alteration in DNA packaging in sperm has the potential to affect the cell's ability to effectively deliver the paternal genome to the egg, and could likely also cause epigenetic regulatory problems in the developing embryo.

\section{PROTAMINE INCORPORATION, HISTONE RETENTION AND INFERTILITY}

Male factor infertility has been linked to aberrant protamination in a number of different studies, all of which have demonstrated anomalies either at the single-cell level or as an entire population. Numerous analytical tools have been developed to determine whether proper chromatin compaction has occurred during spermatogenesis: P1/P2 ratio analysis, histone to protamine ratio analysis and various antibody-staining techniques. ${ }^{33,34}$ Indirect assessment of DNA fragmentation has also proven to correlate strongly with incomplete protamine incorporation. $^{35}$

Studies focusing on $\mathrm{P} 1 / \mathrm{P} 2$ ratio analysis have demonstrated the important role of protamine incorporation into the chromatin and have shown a strong correlation between nuclear proteins and general infertility. ${ }^{33}$ In patients undergoing in vitro fertilization treatment, $\mathrm{P} 1 /$ P2 ratios were shown to correlate significantly with fertilization ability. ${ }^{4}$ This finding was thought to possibly be indicative of other overarching abnormalities in the sperm population as a whole. Low protamine levels and abnormal $\mathrm{P} 1 / \mathrm{P} 2$ ratios in both fertile and infertile men were tightly correlated with DNA fragmentation, demonstrating that aberrant protamination results in a chromatin structure that is more susceptible to DNA damage. ${ }^{35-37}$ Histone to protamine ratios can directly detect aberrations in histone retention and have been found to correlate with infertility in humans. Some classes of infertile men have higher histone to protamine ratios than do fertile men, again suggesting problems in the histone to protamine transitional processing. ${ }^{34,38}$ DNA damage in murine sperm was also correlated with low P2 concentrations. Interestingly, these researchers also observed effects on the embryo by utilizing intra-cytoplasmic sperm injection to fertilize the eggs and found that sperm DNA fragmentation resultant from low P2 concentrations was lethal for the embryo. ${ }^{39}$

These studies demonstrate that the chromatin structure of the paternal genome is vital, although the mechanism for regulatory control in the early embryo has remained unclear until recently. Hammoud et al. ${ }^{29,40}$ recently demonstrated that histone retention occurs at specific developmental sites in the early embryo, which has the potential to poise genes for activation. This provides yet another regulatory mechanism in which paternal DNA is poised for activation at specific sites required for normal embryogenesis, establishing further evidence for its role in developmental regulation.

\section{HISTONE MODIFICATIONS}

Chromatin packaging and structural modifications require the integration and cooperation of multiple different types of nuclear proteins. Of great interest in the field of cell biology, genetics and epigenetics is the role of these proteins not only in structural stabilization/modification in the nucleus, but also in gene regulation. Histone proteins are the most abundant nuclear protein in somatic cells and have the distinct ability to facilitate both gene activation and silencing via modification of lysine $(\mathrm{K})$ and serine $(\mathrm{S})$ residues on tail regions, making them potent epigenetic regulators. In the mature sperm, there is a marked decrease in histone protein concentration as a result of dramatic chromatin structural changes, lending credence to previously held beliefs that sperm lack the ability to drive epigenetic changes in the embryo. Importantly, the structural changes throughout spermatogenesis do not leave the sperm entirely void of nucleosomebound DNA; in fact the small amount of histones retained are likely of great influence in the developing embryo. ${ }^{29,40,41}$

The mammalian histone protein family has essential roles in gametes. Histone $2 \mathrm{~A}$ and $2 \mathrm{~B}(\mathrm{H} 2 \mathrm{~A}$ and $\mathrm{H} 2 \mathrm{~B})$, histone $3(\mathrm{H} 3)$, histone $4(\mathrm{H} 4)$ and testes-specific histone variant (tH2B) all are vital to guide the maturing sperm through spermatogenesis. ${ }^{8,42-46}$ Each of these proteins can influence activation or inhibition of gene transcription based on histone tail modifications including: phosphorylation, methylation, acetylation and ubiquitination. Histone modifications are capable of eliciting different genetic responses individually, or may act in concert with other nearby modified proteins to ensure a proper transcriptional state at specific genes. Activation of genes is facilitated by many different modifications, including acetylation of $\mathrm{H} 3$ and $\mathrm{H} 4$, methylation of $\mathrm{H} 3 \mathrm{~K} 4$ and ubiquitination of $\mathrm{H} 2 \mathrm{~B}$. Genes are driven towards inactivation by methylation of $\mathrm{H} 3 \mathrm{~K} 9$ and $\mathrm{H} 3 \mathrm{~K} 27$, deacetylation of $\mathrm{H} 3$ and $\mathrm{H} 4$, and ubiquitination of $\mathrm{H} 2 \mathrm{~A}^{42,44,45,47}$ Various families of enzymes regulate these modifications. The establishment and removal of acetylation is accomplished by histone acetyltransferases and deacetylases, respectively. ${ }^{42}$ Methylation is regulated by histone methyl-transferase and demethylase families, which exhibit strong specificity toward various histone proteins. ${ }^{47}$

\section{HISTONE TAIL ACETYLATION AND METHYLATION, INFERTILITY AND EMBRYOGENESIS}

Histone tail modifications are an essential component in the epigenetic regulation of transcription in the developing sperm cell as well as in the embryo. To ensure proper progression through the various stages of spermatogenesis, histones undergo transitions in their modification states. This allows for the transient transcription patterns seen at many genes during development. To establish mechanisms of action of histone tail modifications, researchers have observed phenotypes resulting from targeted manipulations. 
Recent literature has demonstrated the importance of the regulation of methylation and acetylation in the developing sperm cell. Disruptions in normal methylation status on histones resulted in varying degrees of fertility loss. These induced abnormalities produced animals with germ cells that have a complete lack of transition protein 1 and $\mathrm{P} 1$ production and a resultant decrease in fertility. ${ }^{8}$ Various degrees of fertility loss, including sterility, were seen with many different treatments, all involving the perturbation of methylation marks on histones. ${ }^{48,49}$ Mice treated with deacetylase inhibitors had significant decreases in the number of mature sperm produced and an overall loss of fertility. ${ }^{46,50}$ These results, taken together, demonstrate the importance of various histone modifications and their influence on spermatogenesis and male fertility.

The ability of histones to drive genes toward active or inactive states provides yet another mechanism by which sperm may have epigenetic effects on the embryo. Any histone incorporation into the paternal genome upon delivery to the egg could provide a degree of regulation during embryogenesis. For this to be true, histone retention would have to be localized at regions important in embryonic development. As mentioned earlier, the areas in which we find nucleosomal retention in the mature sperm are regions of great influence to the embryo: developmental promoters, microRNA clusters and imprinted genes. ${ }^{29,40}$ For these genes to be truly poised for activation, it would require that histone modifications within the nucleosome are those that turn genes toward activation (that is, $\mathrm{H} 3 / \mathrm{H} 4$ acetylation, $\mathrm{H} 3 \mathrm{~K} 4 \mathrm{me}$ and $\mathrm{H} 2 \mathrm{Bub}$ ). This is precisely what is seen at many important developmental gene promoters with increased amounts of H3K4me. ${ }^{29,41}$ Many of these regions of retained activation marks also demonstrate bivalency by containing histone modifications that, if acting alone, inhibit activation (H3K27me). This bivalent expression of histone modifications is a hallmark of totipotency and likely contributes to the totipotent state of embryonic stem cells. ${ }^{51}$ These data suggest that mature spermatozoa are poised epigenetically to contribute to the epigenetic state of the embryo.

\section{THE ROLE OF RNA}

In addition to previously mentioned mechanisms of epigenetic regulation found in sperm, recent studies have suggested a role for RNA transcripts found in mature spermatozoa. ${ }^{52,53}$ Although the contributions of RNA to the epigenetic state of sperm still remain somewhat controversial, some possible mechanisms of action are beginning to take shape. It has been proposed that spermatozoal RNA is likely capable of maintaining nuleosome-bound DNA and preventing protamination in specific chromatin regions. ${ }^{54}$ Retained histones are found in chromatin that lies near the nuclear envelope, the same region in which RNAs are found. This regional association allows for interactions that result in inhibition of the histone to protamine transition. The IGF-2 gene, which is essential in embryonic development, provides an excellent model for RNA involvement in maintenance of nucleosome-retained regions of the chromatin. The specific portion of chromatin that contains the IGF-2 gene and its promoter is located near the nuclear envelope and is a major region of histone retention ${ }^{54,55}$ This localization supports the idea that RNA may facilitate, at least in part, structural maintenance of an open chromatin state in specifically localized regions of DNA. The ability of RNA to affect chromatin structure gives it the distinct capability to directly influence eventual gene activation.

\section{CONCLUSION AND FUTURE DIRECTIONS}

In order for the mature mammalian spermatozoa to provide some degree of regulatory control in the developing embryo requires that important genes are epigenetically poised for activation upon delivery to the oocyte. Poising mechanisms in the male germ cell utilize dynamic chromatin structural changes to confer epigenetic regulation of gene transcription in the embryo, which is of vital importance in the creation of healthy and viable offspring. Three major factors work individually or in concert in the sperm chromatin to elicit targeted gene activation or silencing: DNA methylation (or lack of methylation), nucleosome retention and histone tail modifications. Perturbations in any of these mechanisms will likely result in varying degrees of infertility.

Although much has been discovered in recent years regarding the sperm and its ability to influence embryonic development, we still lack well-defined mechanisms of action. Limited work is available, demonstrating direct links between an abnormal epigenetic state in the sperm and altered embryogenesis; most studies have focused on alterations in spermatogenesis and fertility status. This lack of direct study has left us with questions regarding the sperm's ability to facilitate the safe delivery of paternal DNA to the oocyte that is competent to induce transcriptional activation and inhibition at genes important in embryogenesis. Targeted studies involving abnormal epigenetic states in sperm with correlations in abnormal embryogenesis are required to solidify our understanding of the importance of the epigenetic state of spermatozoa on embryonic development.

\section{COMPETING FINANCIAL INTERESTS}

The authors declare no competing financial interests.

1 La Salle S, Oakes CC, Neaga OR, Bourc'his D, Bestor TH et al. Loss of spermatogonia and wide-spread DNA methylation defects in newborn male mice deficient in DNMT3L. BMC Dev Biol 2007; 7: 104.

2 Balhorn R, Reed S, Tanphaichitr N. Aberrant protamine 1/protamine 2 ratios in sperm of infertile human males. Experientia 1988; 44: 52-5.

3 Oliva R. Protamines and male infertility. Hum Reprod Update 2006; 12: 417-35.

4 Aoki VW, Liu L, Jones KP, Hatasaka HH, Gibson M et al. Sperm protamine 1/protamine 2 ratios are related to in vitro fertilization pregnancy rates and predictive of fertilization ability. Fertil Steril 2006; 86: 1408-15.

5 Kaneda M, Okano M, Hata K, Sado T, Tsujimoto N et al. Essential role for de novo DNA methyltransferase Dnmt3a in paternal and maternal imprinting. Nature 2004; 429: 900-3.

6 Marques CJ, Carvalho F, Sousa M, Barros A. Genomic imprinting in disruptive spermatogenesis. Lancet 2004; 363: 1700-2.

7 Cisneros FJ. DNA methylation and male infertility. Front Biosci 2004; 9: 1189-200.

8 Okada Y, Scott G, Ray MK, Mishina Y, Zhang Y. Histone demethylase JHDM2A is critical for Tnp1 and Prm1 transcription and spermatogenesis. Nature 2007; 450: 119-23.

9 Dadoune JP. The nuclear status of human sperm cells. Micron 1995; 26: 323-45.

10 Oliva R, Dixon GH. Vertebrate protamine gene evolution I. Sequence alignments and gene structure. J Mol Evol 1990; 30: 333-46.

11 Hecht NB. Regulation of 'haploid expressed genes' in male germ cells. J Reprod Fertil 1990; 88: 679-93.

12 Eden S, Cedar H. Role of DNA methylation in the regulation of transcription. Curr Opin Genet Dev 1994; 4: 255-9.

13 Okano M, Bell DW, Haber DA, Li E. DNA methyltransferases Dnmt3a and Dnmt3b are essential for de novo methylation and mammalian development. Cell 1999; 99 : 247 57.

14 Bestor TH. Activation of mammalian DNA methyltransferase by cleavage of a $\mathrm{Zn}$ binding regulatory domain. EMBO J 1992; 11: 2611-7.

$15 \mathrm{Li} \mathrm{E}$, Bestor TH, Jaenisch R. Targeted mutation of the DNA methyltransferase gene results in embryonic lethality. Cell 1992; 69: 915-26.

16 Surani MA. Imprinting and the initiation of gene silencing in the germ line. Cel/ 1998 93: 309-12.

$17 \mathrm{Ng} \mathrm{HH}$, Bird A. DNA methylation and chromatin modification. Curr Opin Genet Dev 1999; 9: 158-63.

18 Panning B, Jaenisch R. DNA hypomethylation can activate Xist expression and silence X-linked genes. Genes Dev 1996; 10: 1991-2002.

19 Walsh CP, Chaillet JR, Bestor TH. Transcription of IAP endogenous retroviruses is constrained by cytosine methylation. Nat Genet 1998; 20: 116-7.

20 Jaenisch R, Jahner D. Methylation, expression and chromosomal position of genes in mammals. Biochim Biophys Acta 1984; 782: 1-9. 
21 Chedin F, Lieber MR, Hsieh CL. The DNA methyltransferase-like protein DNMT3L stimulates de novo methylation by Dnmt3a. Proc Natl Acad Sci USA 2002; 99: $16916-21$

22 Kaneda M, Sado T, Hata K, Okano M, Tsujimoto N et al. Role of de novo DNA methyltransferases in initiation of genomic imprinting and X-chromosome inactivation. Cold Spring Harb Symp Quant Biol 2004; 69: 125-9.

23 Kato Y, Kaneda M, Hata K, Kumaki K, Hisano M et al. Role of the Dnmt3 family in de novo methylation of imprinted and repetitive sequences during male germ cell development in the mouse. Hum Mol Genet 2007; 16: 2272-80.

24 Egger G, Liang G, Aparicio A, Jones PA. Epigenetics in human disease and prospects for epigenetic therapy. Nature 2004; 429: 457-63.

25 Raman R, Narayan G. 5-Aza deoxyCytidine-induced inhibition of differentiation of spermatogonia into spermatocytes in the mouse. Mol Reprod Dev 1995; 42: 284-90.

26 Oakes CC, Kelly TL, Robaire B, Trasler JM. Adverse effects of 5-aza-2'-deoxycytidine on spermatogenesis include reduced sperm function and selective inhibition of de novo DNA methylation. J Pharmacol Exp Ther 2007; 322: 1171-80.

27 Kelly TL, Li E, Trasler JM. 5-aza-2'-deoxycytidine induces alterations in murine spermatogenesis and pregnancy outcome. J Androl 2003; 24: 822-30.

28 Doerksen T, Trasler JM. Developmental exposure of male germ cells to 5-azacytidine results in abnormal preimplantation development in rats. Biol Reprod 1996; 55: $1155-62$.

29 Hammoud SS, Nix DA, Zhang H, Purwar J, Carrell DT et al. Distinctive chromatin in human sperm packages genes for embryo development. Nature 2009; 460: 473-8.

30 Benchaib M, Braun V, Ressnikof D, Lornage J, Durand P et al. Influence of global sperm DNA methylation on IVF results. Hum Reprod 2005; 20: 768-73.

31 Ward WS, Coffey DS. DNA packaging and organization in mammalian spermatozoa: comparison with somatic cells. Biol Reprod 1991; 44: 569-74.

32 Balhorn R. The protamine family of sperm nuclear proteins. Genome Biol 2007; 8: 227.

33 Aoki VW, Liu L, Carrell DT. Identification and evaluation of a novel sperm protamine abnormality in a population of infertile males. Hum Reprod 2005; 20: 1298-306.

34 Zhang X, San Gabriel M, Zini A. Sperm nuclear histone to protamine ratio in fertile and infertile men: evidence of heterogeneous subpopulations of spermatozoa in the ejaculate. J Androl 2006; 27: 414-20.

35 Aoki VW, Moskovtsev SI, Willis J, Liu L, Mullen JB et al. DNA integrity is compromised in protamine-deficient human sperm. J Androl 2005; 26: 741-8.

36 Aoki VW, Emery BR, Liu L, Carrell DT. Protamine levels vary between individual sperm cells of infertile human males and correlate with viability and DNA integrity. J Androl 2006; 27 : 890-8.

37 Torregrosa N, Dominguez-Fandos D, Camejo MI, Shirley CR, Meistrich ML et al. Protamine 2 precursors, protamine 1/protamine 2 ratio, DNA integrity and other sperm parameters in infertile patients. Hum Reprod 2006; 21: 2084-9.

38 Zini A, Gabriel MS, Zhang X. The histone to protamine ratio in human spermatozoa: comparative study of whole and processed semen. Fertil Steril 2007: 87: 217-9.
39 Cho C, Jung- Ha H, Willis WD, Goulding EH, Stein P et al. Protamine 2 deficiency leads to sperm DNA damage and embryo death in mice. Biol Reprod 2003; 69: 211-7.

40 Arpanahi A, Brinkworth M, Iles D, Krawetz SA, Paradowska A et al. Endonucleasesensitive regions of human spermatozoal chromatin are highly enriched in promoter and CTCF binding sequences. Genome Res 2009; 19: 1338-49.

41 Brykczynska U, Hisano M, Erkek S, Ramos L, Oakeley EJ et al. Repressive and active histone methylation mark distinct promoters in human and mouse spermatozoa. Nat Struct Mol Biol 2010; 17: 679-87.

42 Jenuwein T, Allis CD. Translating the histone code. Science $2001 ; 293: 1074-80$.

43 Gatewood JM, Cook GR, Balhorn R, Schmid CW, Bradbury EM. Isolation of four core histones from human sperm chromatin representing a minor subset of somatic histones. J Biol Chem 1990; 265: 20662-6.

44 Zhu B, Zheng $Y$, Pham AD, Mandal SS, Erdjument-Bromage $\mathrm{H}$ et al. Monoubiquitination of human histone $\mathrm{H} 2 \mathrm{~B}$ : the factors involved and their roles in HOX gene regulation. $\mathrm{Mol}$ Cell 2005; 20: 601-11.

45 Baarends WM, Wassenaar E, van der Laan R, Hoogerbrugge J, Sleddens-Linkels E et al. Silencing of unpaired chromatin and histone $\mathrm{H} 2 \mathrm{~A}$ ubiquitination in mammalian meiosis. Mol Cell Biol 2005; 25: 1041-53.

46 Fenic I, Sonnack V, Failing K, Bergmann M, Steger K. In vivo effects of histonedeacetylase inhibitor trichostatin-A on murine spermatogenesis. J Androl 2004; 25: 811-8.

47 Lachner M, Jenuwein T. The many faces of histone lysine methylation. Curr Opin Cell Biol 2002; 14: 286-98.

48 Glaser S, Lubitz S, Loveland KL, Ohbo K, Robb L et al. The histone 3 lysine 4 methyltransferase, MII2, is only required briefly in development and spermatogenesis. Epigenetics Chromatin 2009; 2: 5 .

49 Lee MG, Wynder C, Cooch N, Shiekhattar R. An essential role for CoREST in nucleosomal histone 3 lysine 4 demethylation. Nature 2005; 437: 432-5.

50 Fenic I, Hossain HM, Sonnack V, Tchatalbachev S, Thierer F et al. In vivo application of histone deacetylase inhibitor trichostatin-a impairs murine male meiosis. J Androl 2008; 29: 172-85

51 Bernstein BE, Mikkelsen TS, Xie X, Kamal M, Huebert DJ et al. A bivalent chromatin structure marks key developmental genes in embryonic stem cells. Cell 2006; 125: 315-26.

52 Rassoulzadegan M, Grandjean V, Gounon P, Vincent S, Gillot I et al. RNA-mediated non-mendelian inheritance of an epigenetic change in the mouse. Nature 2006; 441: 469-74.

53 Dadoune JP. Spermatozoal RNAs: what about their functions? Microsc Res Tech 2009; 72: 536-51.

54 Miller D, Ostermeier GC, Krawetz SA. The controversy, potential and roles of spermatozoal RNA. Trends Mol Med 2005; 11: 156-63.

55 Wykes SM, Krawetz SA. The structural organization of sperm chromatin. J Biol Chem 2003; 278: 29471-7. 